\title{
Finite size effects and the supersymmetric sine-Gordon models
}

\author{
Clare Dunning ${ }^{1}$ \\ Dept. of Mathematics, University of York, York YO1 5DD, UK
}

\begin{abstract}
We propose nonlinear integral equations to describe the groundstate energy of the fractional supersymmetric sine-Gordon models. The equations encompass the $N=1$ supersymmetric sine-Gordon model as well as the $\phi_{\text {id,id,adj }}$ perturbation of the $S U(2)_{L} \times S U(2)_{K} / S U(2)_{L+K}$ models at rational level $K$. A second set of equations are proposed for the groundstate energy of the $N=2$ supersymmetric sine-Gordon model.
\end{abstract}

\section{Introduction}

The sine-Gordon (SG) and supersymmetric sine-Gordon (SSG) [1, 2, 3, 4, 5, models belong to an interesting set of integrable quantum field theories that are said to have fractional supersymmetry 6 6], thus attracting the name fractional supersymmetric sine-Gordon models (FSSG). The field content [8, 9] consists of a single boson interacting with a $Z_{L}$ generating parafermion, the choices $L=1$ and 2 yielding the SG and SSG models respectively. The models correspond to the fractional conformal field theory obtained from the Wess-ZuminoWitten model $S U(2)_{L}$ by compactifying the free boson on a circle of radius $2 R=\beta / \sqrt{4 \pi}$, perturbed by

$$
\Phi(z, \bar{z})=\frac{g \sqrt{4 \pi}}{\beta} \Psi_{1}(z) \bar{\Psi}_{1}(z) e^{-i \frac{\beta}{\sqrt{4} \pi} \varphi(z, \bar{z})}+\text { c.c. } .
$$

The mass scale is set by the dimensionful parameter $g$, and the real dimensionless parameter $\beta$ determines the particle content. Since the boson $\varphi(z, \bar{z})$ and generating parafermion $\Psi_{1}(z, \bar{z})$ have conformal dimensions 1 and $1-1 / L$ respectively, the perturbing term has conformal dimension $\beta^{2} / 8 \pi+1-1 / L$. The ultraviolet effective central charge is $1+2(L-1) /(L+2)=$ $3 L / L+2$.

In the limit $\beta^{2} / 8 \pi \rightarrow 1 / L$ the perturbed model becomes the current-current perturbation of the WZW model $S U(2)_{L}[8]$. Instead if we tune the parameter

$$
\xi=\frac{L \beta^{2} / 8 \pi}{1 / L-\beta^{2} / 8 \pi}
$$

to $K+2$ we obtain the unitary coset $S U(2)_{L} \times S U(2)_{K} / S U(2)_{L+K}$ perturbed by the operator

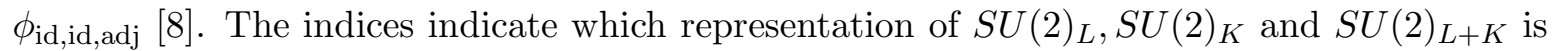
associated to the corresponding weights. This choice of $\xi$ when $L=1$ corresponds to the well-known quantum group restriction [10 11] of the sine-Gordon model to the $\phi_{13}$ perturbed minimal models. In fact, the FSSG models were first proposed as the models obtained by 'unrestricting' the S-matrices describing the $\phi_{\text {id,id,adj }}$ perturbation of the cosets $\mathcal{M}_{L, K} \equiv$ $S U(2)_{L} \times S U(2)_{K} / S U(2)_{L+K}[8$.

The existence of higher spin conserved charges in $1+1$ dimensional integrable quantum field theories strongly constrain the allowed scattering processes. There is no particle production, the individual particle momenta are conserved, and all $n$-particle scattering processes

\footnotetext{
${ }^{1}$ tcd1@york.ac.uk
} 
decompose into products of two-particle scattering amplitudes [12. The S-matrix of a supersymmetric model should also commute with the supersymmetry generators. This typically leads to a further factorisation of the two-particle S-matrix into a direct product in which one factor encodes the supersymmetry dependence [8, 13, 9]. The S-matrices of the FSSG models exactly fit this pattern [8, 9]:

$$
S(\beta)=S_{L} \otimes S_{\mathrm{SG}}(\beta) .
$$

The supersymmetry-related factor $S_{L}$ describes the scattering of the massive $\phi_{13}$ perturbation of the minimal model $\mathcal{M}_{1, L}, 7,11,6$, and the bosonic factor $S_{\mathrm{SG}}(\beta)$ is the S-matrix of the sine-Gordon model at coupling $\beta$ [12]. The particle content of the FSSG consists of a soliton of mass $M$ and its antisoliton together with a number of breathers of mass

$$
M_{j}=2 M \sin \left(\frac{\pi j \xi}{2}\right) \quad, \quad j=1 \ldots<\frac{1}{\xi} .
$$

There are no breathers in the repulsive regime $\xi<1$. Once the spectrum and associated Smatrices have been conjectured the thermodynamic Bethe ansatz [14,15] is usually employed to check the proposed scattering theory. However when the scattering is nondiagonal, as above, deriving the TBA equations from the S-matrices becomes rather difficult.

Fendley and Intriligator noted that one can immediately write down a TBA system for a model with a tensor product S-matrix of the form $S_{G} \otimes S_{H}$ if the TBA equations are already known for the models described by S-matrices $S_{G}$ and $S_{H}$ [16]. If the individual TBA equations are encoded on Dynkin-like diagrams of type $G$ and $H$, each having a single massive node, then the TBA equations for the model with S-matrix $S_{G} \otimes S_{H}$ are found by gluing the individual TBA equations at the massive node [16]. In the compact notation of [17, 18, this corresponds to $(G \diamond H)_{L}$, where $L$ indicates the massive node. It is easy to check that the TBA equations found for the FSSG model at the special values of the coupling [16, 19, 17, 20, 21]

$$
\frac{\beta^{2}}{8 \pi}=\frac{1}{L}-\frac{2}{m} \quad, \quad m \in \mathbb{N}^{+} \quad, \quad L=1,2, \ldots \leq \operatorname{int}[m / 2]-1,
$$

and those for the $\phi_{\text {id,id,adj }}$ perturbation of the $\mathcal{M}_{L, K}$ models [22] all fit this pattern. TBA equations for the models arising as the $\Phi_{13}$ perturbation of the supersymmetric models $\mathcal{S M}_{2,4 n+4}$ are known [23.24.25 26], but currently lack an interpretation in terms of Dynkin-like diagrams.

A $Y$-system, from which the TBA equations can be obtained via Fourier transform [27, for the FSSG model at any coupling has been proposed using the gluing idea and the sineGordon $Y$-system 20,21,28. The SG $Y$-system depends on writing the smaller of $\xi$ or $1 / \xi$ as a simple continued fraction. The number of TBA equations is found to be equal to the sum of the leading terms of the denominators in the continued fraction plus one further equation. Though there is an neat depiction in terms of Dynkin diagrams 20, 21] it is hard to deal with a large number of equations. Moreover those $\xi$ for which the continued fraction does not terminate are associated to a TBA system with an infinite number of equations.

There is an alternative method of calculating the finite size effects of the sine-Gordon model, known as the nonlinear integral equation (NLIE) technique. The result is a single equation, first proposed for the groundstate of the sine-Gordon model by Destri and De Vega in 1992 [29]. A complete derivation to include the excited states of even topological charge followed in [30], and an equation for the excited states of odd topological charge was proposed in 31. The conformal limit of the groundstate equation had been proposed in 1991 in an integrable lattice model context by Klümper, Batchelor and Pearce 33 32. Further motivation for this work came from the the lattice model connection: the $X X X$ spin chain at spin $S$ is a model with underlying field theory $S U(2)_{2 S}$. The spin- $\frac{1}{2}$ case corresponds to the lattice model 
mentioned above; the spin-1 problem is discussed in 32,33. Recently Suzuki has generalised the technique of [33 32] to obtain a a finite set of nonlinear integral equations that describe the thermodynamics of the spin- $S$ chain at finite temperature 34. Given the relations between the spin chains and $S U(2)_{2 S}$, and the FSSG models and perturbed $S U(2)_{L}$, we should expect the conformal limit of any integral equation describing the FSSG at $\beta^{2}=1 / L$ to be closely related to the zero temperature version of Suzuki's NLIE.

Motivated by Suzuki's equations and the gluing idea we propose a set of nonlinear integral equations to describe the groundstate energy of the fractional supersymmetric sine-Gordon model at any value of the coupling constant $\beta^{2}$. In $\S 2$ a short description of the component equations leads to the proposed NLIEs, and then a number of tests are carried out in $\S 3$.

The $N=2$ supersymmetric sine-Gordon model is not a member of the FSSG series. However the groundstate energy of this model can be studied by means of a further set of NLIEs, obtained by a simple generalisation of the FSSG equations. The details are in $\S 4$ along with a short discussion of related models: the sausage models and the parafermionic perturbation of the $Z_{M}$ models. Directions for future work can be found in $\S 5$.

\section{Nonlinear integral equations}

There are two integrable quantum field theories associated with the $\phi_{13}$ perturbation of the minimal model $\mathcal{M}_{1, L}$. If the coupling $\lambda$ is positive the action

$$
\mathcal{A}=\mathcal{A}_{\mathrm{CFT}}+\lambda \int d^{2} x \phi_{13}
$$

describes a 'massless flow', or renormalisation group trajectory, from $\mathcal{M}_{1, L}$ in the ultraviolet to $\mathcal{M}_{1, L-1}$ in the infrared [35]. On the other hand, if the coupling is negative ([6) describes a massive integrable quantum field theory with S-matrix $S_{L}$ [36]. The TBA equations for the groundstate energy of the massive scattering theory were found by Al.B. Zamolodchikov [37. As we explain below one small change allows the same equations to unexpectedly describe the groundstate energy of the massless flows [38. The equations are written in terms of the incidence matrix $I_{a b}$ of the $A_{L}$ Dynkin diagram [37, 38]. The TBA system is*

$$
\begin{aligned}
f_{a}(\theta) & =g_{a}(\theta)+\sum_{b=1}^{L} \int_{-\infty}^{\infty} d \theta^{\prime} I_{a b} \chi\left(\theta-\theta^{\prime}\right) \ln \left(1+e^{f_{b}\left(\theta^{\prime}\right)}\right) \\
c_{\mathrm{eff}}(r) & =\frac{6}{\pi} \sum_{a=1}^{L} \int_{-\infty}^{\infty} d \theta g_{a}(\theta) \ln \left(1+e^{f_{a}(\theta)}\right),
\end{aligned}
$$

with kernel $\chi(\theta)=1 /(2 \pi \cosh \theta)$. Here $r=M R$ where $M$ is the mass of the elementary kink, or in the massless theory the crossover scale, and $R$ is the circumference of the cylinder on which the model is considered. The massive theory is found by setting the driving term to

$$
g_{a}(\theta)=-\delta_{a, 1} r \cosh \theta
$$

while the massless theory appears if

$$
g_{a}(\theta)=-\frac{r}{2}\left(\delta_{a, L} e^{\theta}+\delta_{a, 1} e^{-\theta}\right) .
$$

\footnotetext{
${ }^{*}$ Note that the TBA equations are usually written in terms of pseudoenergy $\varepsilon=-f$.
} 
Both theories have the same ultraviolet effective central charge: $c_{\text {eff }}(0)=1-6 /(L+2)(L+3)$, while the infrared value is zero or $1-6 /(L+1)(L+2)$ for the massive/massless models respectively.

To avoid dealing with a different and possibly infinite set of equations for each choice of $\beta^{2}$ we consider the second type of nonlinear integral equation mentioned in the introduction. The equation involves a complex function $a(\theta)$ and its conjugate $\bar{a}(\theta)$ coupled via $33,32,29$,

$$
\ln a(\theta)=-i r \sinh \theta+\int_{\mathcal{C}_{1}} d \theta^{\prime} \varphi\left(\theta-\theta^{\prime}\right) \ln \left(1+a\left(\theta^{\prime}\right)\right)-\int_{\mathcal{C}_{2}} d \theta^{\prime} \varphi\left(\theta-\theta^{\prime}\right) \ln \left(1+a^{-1}\left(\theta^{\prime}\right)\right)
$$

The integration contours $\mathcal{C}_{1}$ and $\mathcal{C}_{2}$ run from $-\infty$ to $+\infty$, just below and just above the real $\theta$-axis respectively. The kernel $\varphi(\theta)$ is proportional to the logarithmic derivative of the soliton-soliton scattering amplitude of the sine-Gordon model

$$
\varphi(\theta)=\int \frac{d k}{2 \pi} e^{i k \theta} \frac{\sinh (\xi-1) \frac{\pi k}{2}}{2 \sinh \frac{\pi \xi k}{2} \cosh \frac{\pi k}{2}} .
$$

The effective central charge found using

$$
c_{\mathrm{eff}}(r)=\frac{3 i r}{\pi^{2}}\left(\int_{\mathcal{C}_{1}} d \theta \sinh \theta \ln (1+a(\theta))-\int_{\mathcal{C}_{2}} d \theta \sinh \theta \ln \left(1+a^{-1}(\theta)\right)\right)
$$

has ultraviolet value 1 . With constant $i \pi \alpha$ [39] added to the RHS of (10) the effective central charge becomes $c_{\text {eff }}(0)=1-6 \alpha^{2} \xi /(\xi+1)$. The choice $\xi=p /(q-p)$ and $\alpha= \pm 1 / p$ [0, 41, 42] implements the restriction [10,11] of the SG model to the $\phi_{13}$ perturbed minimal models.

The explanation for Fendley and Intriligator's gluing proposal lies in the derivation of the TBA equations [16. The key is the factorisability of the S-matrix: it implies each factor can be diagonalised separately and each generates an individual set of pseudoparticles. These are seen to only couple through the massive particle, with the result being the 'factorised' or glued TBA system described in the introduction. There is no equivalent of the pseudoparticles in the derivation of the sine-Gordon NLIE. Moreover, in further contrast to the TBA, the Smatrix plays no role in the NLIE derivation. Instead one notes a posteriori that the kernel function of the resulting NLIE is related as described above to the sine-Gordon S-matrix. It therefore seemed unlikely that the gluing idea would be of any use in finding a NLIE for a model with an S-matrix of the form (3). Fortunately this turns out not to be the case.

We obtain the NLIEs for the FSSG model by gluing the minimal model TBA equations to the sine-Gordon NLIE at the massive node following the Fendley-Intriligator prescription [16. However the resulting equations are not quite right and it is necessary to make a few simple modifications. Due to singularities in $\ln \left(1+y_{1}(\theta)\right)$ for real $\theta$ a TBA-like convolution $\int d \theta^{\prime} \chi\left(\theta-\theta^{\prime}\right) \ln \left(1+y_{1}\left(\theta^{\prime}\right)\right)$ along the real axis cannot be used. The solution is to replace this term with a convolution along the contours $\mathcal{C}_{1}$ and $\mathcal{C}_{2}$ encircling the real axis as in the SG NLIE. To ensure for real $\theta$ the reality of the $L-1$ TBA-like functions $y_{2}, \ldots, y_{L}$, and the complex nature of the NLIE-like function $y_{1}$ we find we should couple $y_{1}$ and $y_{2}$ to each other with an extra shift of $i \pi / 2$ in the kernel $\chi$. The conjectured equations are

$$
\begin{aligned}
\ln y_{1}(\theta)=\nu_{1}(\theta) & +i \pi \alpha \\
& +\int_{\mathcal{C}_{1}} d \theta^{\prime} \varphi\left(\theta-\theta^{\prime}\right) \ln \left(1+y_{1}\left(\theta^{\prime}\right)\right)-\int_{\mathcal{C}_{2}} d \theta^{\prime} \varphi\left(\theta-\theta^{\prime}\right) \ln \left(1+y_{1}^{-1}\left(\theta^{\prime}\right)\right) \\
& \quad+\int_{-\infty}^{\infty} d \theta^{\prime} \chi\left(\theta-\theta^{\prime}+\frac{i \pi}{2}\right) \ln \left(1+y_{2}\left(\theta^{\prime}\right)\right)
\end{aligned}
$$




$$
\begin{aligned}
& \ln y_{2}(\theta)=\nu_{2}(\theta)+\int_{\mathcal{C}_{1}} d \theta^{\prime} \chi\left(\theta-\theta^{\prime}-\frac{i \pi}{2}\right) \ln \left(1+y_{1}\left(\theta^{\prime}\right)\right)-\int_{\mathcal{C}_{2}} d \theta^{\prime} \chi\left(\theta-\theta^{\prime}-\frac{i \pi}{2}\right) \ln \left(1+y_{1}^{-1}\left(\theta^{\prime}\right)\right) \\
&+\int_{-\infty}^{\infty} d \theta^{\prime} \chi\left(\theta-\theta^{\prime}\right) \ln \left(1+y_{3}\left(\theta^{\prime}\right)\right) ; \\
& \ln y_{a}(\theta)=\nu_{a}(\theta)+\sum_{b=1}^{L} I_{a b} \int_{-\infty}^{\infty} d \theta^{\prime} \chi\left(\theta-\theta^{\prime}\right) \ln \left(1+y_{b}\left(\theta^{\prime}\right)\right) \quad, \quad a=3 \ldots L
\end{aligned}
$$

where $\chi(\theta)$ remains as above. The driving terms are

$$
\nu_{a}(\theta)=\left\{\begin{array}{cl}
-i r \sinh (\theta) & a=1 \\
0 & \text { otherwise }
\end{array} .\right.
$$

Borrowing the TBA notation of [17, 18, our shorthand for equations of this type will be $\left(A_{1} \diamond A_{L}\right)_{a}^{[i]}$. The superscript $[i]$ indicates the NLIE-like function and the subscript $a$ the function on which the mass is placed. The equations above are therefore represented by $\left(A_{1} \diamond A_{L}\right)_{1}^{[1]}$. The effective central charge defined in terms of the solution of (13) can be calculated using

$$
c_{\text {eff }}(r)=-\frac{3}{\pi^{2}} \sum_{a=1}^{L} \int_{\mathcal{C}_{1}} d \theta \nu_{a}(\theta) \ln \left(1+y_{a}(\theta)\right)-\int_{\mathcal{C}_{2}} d \theta \nu_{a}(\theta) \ln \left(1+y_{a}^{-1}(\theta)\right) .
$$

At $L=1$ the system (131415) trivially reduces to the NLIE describing the sine-Gordon equation. As expected these equations become equivalent to Suzuki's 34 for the spin- $S$ $X X X$ spin chain in the limit $\beta^{2} \rightarrow 1 / L(\xi \rightarrow \infty)$. The NLIE-like functions are approximately related via $y_{1}(\theta) \approx \ln \mathfrak{a}\left(\frac{2}{\pi} \theta+i\right)$, provided the spin chain driving term is replaced with the one relevant for the field theory.

Based on previous experience with NLIEs we expect to find the effective central charge for the FSSG model at level $L$ and coupling $\beta^{2}$ by tuning $\xi$ according to (2) and setting $\alpha=0$. The $\phi_{\text {id,id,adj }}$ perturbation of the coset $\mathcal{M}_{L, K}$ should be found at

$$
\xi=K+2 \quad, \quad \alpha= \pm 1 /(K+2) .
$$

In fact we hope the equations go a little further: even though the quantum group restriction of the S-matrices may not work it is thought that the FSSG models also describe the nonunitary cosets perturbed by $\phi_{\text {id,id,adj. If we set }}$

$$
\xi=\frac{L p}{q-p} \quad, \quad \alpha= \pm \frac{1}{p},
$$

then the NLIEs should yield the effective central charge of the $\phi_{\text {id,id,adj }}$ perturbation of $\mathcal{M}_{L, L p /(q-p)-2}$. This is a coset at rational, and possibly negative, level. These models make sense provided the integers $p, q$ are such that $p$ and $(q-p) / L$ are coprime integers. At $L=1$ this is the $\phi_{13}$ perturbation of the minimal models $\mathcal{M}(p, q)$, and at $L=2$ the supersymmetry preserving perturbation $\Phi_{13}$ of the superminimal models $\mathcal{S} \mathcal{M}(p, q)$.

In the same way that TBA equations for the massive perturbation of the minimal models can be converted into those describing the massless perturbations, we can describe some massless flows using the proposed NLIEs with new driving terms

$$
\nu_{a}(\theta)=\left\{\begin{array}{ll}
-\frac{i}{2} r e^{\theta} & a=1 \\
-\frac{1}{2} r e^{-\theta} \delta_{a, L-K+1} & \text { otherwise }
\end{array} .\right.
$$




\begin{tabular}{c|c|c|c|c} 
& $a$ & $1+x_{a}^{-}$ & $1+x_{a}^{0}$ & $1+x_{a}^{+}$ \\
\hline Massive & 1 & 1 & $\frac{\sin \frac{\pi \xi \alpha(L+1)}{L+\xi}}{\sin \frac{\pi \xi}{L+\xi}} e^{i \pi \xi \alpha L /(L+\xi)}$ & 1 \\
\hline \multirow{2}{*}{ Massless } & $2 \ldots L$ & $\frac{\sin ^{2} \frac{\pi a}{L+2}}{\sin ^{2} \frac{\pi}{L+2}}$ & $\frac{\sin ^{2} \frac{\pi \xi \alpha(L+2-a)}{L+\xi}}{\sin ^{2} \frac{\pi \xi \alpha}{L+\xi}}$ & $\frac{\sin ^{2} \frac{\pi a}{L+2}}{\sin ^{2} \frac{\pi}{L+2}}$ \\
\hline & $2 \ldots L-K$ & $\frac{\sin \frac{\pi(K-L)}{L+2}}{\sin \frac{\pi}{L+2}} e^{i \pi(K+1) /(L+2)}$ & $\frac{\sin \frac{\pi(L+1)}{L+K+2}}{\sin \frac{\pi}{L+K+2}} e^{i \pi L /(L+K+2)}$ & 1 \\
& $L-K+1 \ldots L$ & $\frac{\sin ^{2} \frac{\pi(K+a)}{L+2}}{\sin ^{2} \frac{\pi}{L+2}}$ & $\frac{\sin ^{2} \frac{\pi(L+2-a)}{L+K+2}}{\sin ^{2} \frac{\pi(L+2-a)}{K+K+2}}$ & $\frac{\sin ^{2} \frac{\pi a}{L+2}}{\sin ^{2} \frac{\pi}{K+2}}$ \\
$\sin ^{2} \frac{\pi}{L+2}$ \\
$\frac{\sin ^{2} \frac{\pi(L+2-a)}{L+K+2}}{\sin ^{2} \frac{\pi a}{L+K+2}}$ & $\frac{\sin ^{2} \frac{\pi a}{L+2}}{\sin ^{2} \frac{\pi}{L+2}}$
\end{tabular}

Table 1: Stationary values for massive and massless perturbed models.

If $(\xi, \alpha)$ are fixed to the values prescribed in (16) the equations reproduce the flow $\mathcal{M}_{L, K}+$ $\phi_{\text {id,id,adj }} \rightarrow \mathcal{M}_{L, K-L}$. Given the different nature of $y_{1}$ and $y_{L-K+1}$ it is rather surprising that the resulting NLIEs do in fact describe massless flows. The massless flows within the sineGordon model $(L=1)$ can alternatively be studied via two coupled NLIE functions 39,43].

Using the symmetry between $L$ and $K$ we can minimise the number of NLIEs for the coset models $\mathcal{M}_{L, K}$ by choosing $L<K$. However the massless flows only make sense if $L>K$. Both possibilities lead to a smaller number of equations than the relevant TBA equations. Therefore the new NLIEs are a computationally more efficient way of calculating the finite size effects of the FSSG models. In the next section we take a closer at the equations and obtain some results confirming the above conjectures.

\section{Testing the NLIEs}

We begin by examining the behaviour of the individual functions at $r=0$ and $r=\infty$. By differentiating the first equation of (13) with respect to $\theta$ we see the function $i \ln \left(y_{1}\right)(\theta)$ becomes approximately constant in the region $|\theta| \ll \ln (2 / r)$ as $r \rightarrow 0$. For $\theta \ll-\ln (2 / r)$ it behaves as $-e^{r \theta}$, and for $\theta \gg \ln (2 / r)$ as $e^{r \theta}$. Therefore $y_{1}$ is a complex constant, which we denote $x_{1}^{0}$, inside the the central region $-\ln (2 / r) \ll \theta \ll \ln (2 / r)$. The real and imaginary parts of $y_{1}$ oscillate outside this region but because $\eta$ is nonzero $y_{1}(\theta-i \eta)$ and $y_{1}^{-1}(\theta+i \eta)$ ultimately tend to zero. The functions $y_{2}, y_{3}, \ldots, y_{L}$ behave exactly as if they were TBA Y-functions ${ }^{\dagger}$ : they are symmetric in $\theta$ and real for real $\theta$. From the behaviour of $y_{1}$ it follows that in the ultraviolet limit they become constants $x_{a}^{-}, x_{a}^{0}, x_{a}^{+}$in the regions $\theta \ll-\ln (2 / r),-\ln (2 / r) \ll$ $\theta \ll \ln (2 / r)$ and $\theta \gg \ln (2 / r)$ respectively, while interpolating smoothly in between. A similar conclusion can be reached for the stationary solutions of the massless equations, apart from a small change in the behaviour of $y_{1}$ as it now becomes a nonzero constant in the region $\theta \ll-\ln (2 / r)$. It is simple to check this picture by plotting some numerical solutions to the NLIEs. The exact values of the stationary solutions, derived from the NLIEs in the usual way [15, 44, are summarised for both massive and massless cases in table 1.

Using the standard methods [15,44,32] we find the ultraviolet effective central charge takes the form

$$
c_{\mathrm{eff}}(0)=-\frac{3}{\pi^{2}}\left[2 \mathcal{L}\left(\frac{1}{1+\bar{x}_{1}^{0}}\right)-\mathcal{L}\left(\frac{1}{1+\bar{x}_{1}^{+}}\right)-\mathcal{L}\left(\frac{1}{1+\bar{x}_{1}^{-}}\right)\right.
$$

\footnotetext{
${ }^{\dagger}$ in standard notation $Y_{a}(\theta)=\exp \left[\varepsilon_{a}(\theta)\right]$
} 


$$
\begin{aligned}
& +\sum_{a=1}^{L} 2 \mathcal{L}\left(\frac{1}{1+x_{a}^{0}}\right)-\mathcal{L}\left(\frac{1}{1+x_{a}^{+}}\right)-\mathcal{L}\left(\frac{1}{1+x_{a}^{-}}\right) \\
& \left.\quad-\frac{i \pi \alpha}{2}\left(\ln \frac{\left(1+x_{1}^{0}\right)^{2}}{\left(1+x_{1}^{-}\right)\left(1+x_{1}^{+}\right)}-\ln \frac{\left(1+\bar{x}_{1}^{0}\right)^{2}}{\left(1+\bar{x}_{1}^{-}\right)\left(1+\bar{x}_{1}^{+}\right)}\right)\right]
\end{aligned}
$$

where $\mathcal{L}$ is Rogers dilogarithm function

$$
\mathcal{L}(x)=-\frac{1}{2} \int_{0}^{x} d t \frac{\ln 1-t}{t}+\frac{\ln t}{1-t} .
$$

To evaluate (19) we use the standard properties and known sum rules of the dilogarithm (see for example [45])

$$
\frac{6}{\pi^{2}} \sum_{a=2}^{L} \mathcal{L}\left(\frac{\sin ^{2}[\pi /(L+2)]}{\sin ^{2}[\pi a /(L+2)]}\right)=\frac{2(L-1)}{L+2} \quad, \quad \frac{6}{\pi^{2}} \mathcal{L}(1)=1,
$$

and one further sum rule which involves both real and complex arguments

$$
\frac{6}{\pi^{2}}\left(\mathcal{L}\left(\frac{1}{1+\bar{x}_{1}^{0}}\right)+\sum_{a=1}^{L} \mathcal{L}\left(\frac{1}{1+x_{a}^{0}}\right)\right)=1
$$

Numerical calculations confirm that (22) holds for arbitrary real numbers $\xi$ and $\alpha, L$ is by definition an integer. The ultraviolet effective central charge predicted from the NLIEs takes the form

$$
c_{\mathrm{eff}}(0)=\frac{3 L}{L+2}-\frac{6 \alpha^{2} \xi L}{L+\xi} .
$$

Does this reproduce the expected results? Yes, it does: with $\xi$ related to $\beta^{2}$ as in (2) and $\alpha=0$, the formula predicts $c_{\text {eff }}(0)=3 L /(L+2)$, whereas the choice (16) leads to the effective central charge of the unitary coset models $\mathcal{M}_{L, K}$ :

$$
c_{\text {eff }}(0)=\frac{3 L}{L+2}-\frac{6 L}{(L+K+2)(K+2)},
$$

and the possibility (17) yields the effective central charge for the nonunitary coset models

$$
c_{\mathrm{eff}}(r)=\frac{3 L}{L+2}-\frac{6 L}{p q} .
$$

If we use the massless driving terms, the individual parts of (19) combine in a different way, but the final result is again (23). Since we have fixed the parameters $\xi=K+2$ and $\alpha= \pm 1 /(K+2)$ we actually obtain (24). We need to check the infrared limit to be sure the NLIEs really do describe these massless flows. As $R \rightarrow \infty$ the contribution to $c_{\text {eff }}(\infty)$ from $y_{L+\xi+3}, \ldots, y_{L}$ becomes vanishingly small. The remaining functions have stationary solutions given by the massless ones in table 1 but with $L$ replaced by $L-K$. Plugging these values into (19) gives

$$
c_{\mathrm{eff}}(\infty)=\frac{3 L}{L+2}-\frac{6 L}{(K+2)(K-L+2)},
$$

which is exactly the ultraviolet effective central charge of $\mathcal{M}_{L, K-L}$.

Before obtaining the conformal dimension of the perturbing operator we comment on a surprising relation between the NLIEs at the coset points and the associated TBA equations. The massive $\phi_{\text {id,id,adj }}$ perturbation of $\mathcal{M}_{L, K}$ has a TBA system based on $\left(A_{1} \diamond A_{L+K-1}\right)_{L}$. The 
symmetry between $L$ and $K$ means that we can either take a NLIE with $L$ functions (below denoted $y_{a}$ ) and $\xi=K+2$, or one with $K$ functions (denoted $\tilde{y}_{a}$ ) and $\xi=L+2$, in either case $c_{\text {eff }}(r)$ is exactly the same. Curiously the magnonic parts of both NLIEs are closely related to the TBA functions. Numerical evidence indicates

$$
\exp \left(f_{a}\right)=\left\{\begin{array}{ll}
y_{L-a+1} & a=1, \ldots, L-1 \\
\tilde{y}_{a-K+1} & a=L+1, \ldots, L+K-1
\end{array} .\right.
$$

The functions with nonzero driving term, $\exp f_{L}, y_{1}, \tilde{y}_{1}$, are not equal, but the integral equations imply they must be related. One such relation is

$$
\begin{aligned}
& \int_{-\infty}^{\infty} d \theta^{\prime} \chi\left(\theta-\theta^{\prime}\right) \ln \left(1+e^{f_{L-2}\left(\theta^{\prime}\right)}\right)= \\
& \int_{\mathcal{C}_{1}} d \theta^{\prime} \chi\left(\theta-\theta^{\prime}-\frac{i \pi}{2}\right) \ln \left(1+y_{1}\left(\theta^{\prime}\right)\right)-\int_{\mathcal{C}_{2}} d \theta^{\prime} \chi\left(\theta-\theta^{\prime}-\frac{i \pi}{2}\right) \ln \left(1+y_{1}^{-1}\left(\theta^{\prime}\right)\right) .
\end{aligned}
$$

The question of the conformal dimension of the perturbing operator, predicted as usual from the periodicity of the functions entering the NLIEs [27, can be partially answered via the relation described above with the coset TBA systems. For $\xi=K+2$ and $\alpha= \pm 1 /(K+2)$ the periodicity of the TBA-like functions $y_{2}, \ldots, y_{L}$ inferred from that of the TBA systems for the $\phi_{\text {id,id,adj }}$ perturbation of the coset $\mathcal{M}_{L, K}$ is $i \pi(\xi+L)$ [22]. It is not expected to depend on $\alpha$ and so one only needs to check what happens at other values of $\xi$. Doing so numerically leads us to believe all of the entire functions $y_{a}(\theta)$ have periodicity $i \pi(\xi+L)$, and therefore a Laurent expansion in powers of $\exp (2 /(\xi+L) \theta)$. The quantities $\ln \left(1+y_{a}(\theta)\right)$ appearing in the kernels of the NLIEs have essentially the same shape as the quantities $\ln \left(1+\exp \left(-\varepsilon_{a}(\theta)\right)\right.$ that appear in a pure TBA system, that is a central plateau which quickly goes to zero for $\theta$ outside the region $|\theta| \ll \ln (2 / r)$. Therefore we may employ Zamolodchikov's argument 27] to predict that $c_{\text {eff }}(r)$ expands in powers of $r^{4 /(\xi+L)}$. Note that for some $\alpha, x_{1}^{0}=0$ and Zamolodchikov's argument acquires a small modification, as explained in [46], with the result that $c_{\text {eff }}(r)$ instead expands in powers of $r^{2 /(\xi+L)}$. However for the choices of $\alpha$ needed here $x_{1}^{0}$ is never identically zero. If the perturbing operator is odd we would expect an expansion in $r^{2(1-\Delta)}$ leading to the prediction $\Delta=1-2 /(\xi+L)$. Inserting the appropriate value of $\xi$ (17) we find $\Delta=1-2 /(K+L+2)$, which exactly matches the conformal dimension of $\phi_{\text {id,id,adj }}$ for both unitary and nonunitary (with $K=L p /(q-p)$ ) cosets. On the other hand if the perturbing operator is even we would expect an expansion in $r^{4(1-\Delta)}$ which implies $\Delta=1-1 /(\xi+L)$. Writing $\xi$ in terms of $\beta^{2}$ using (2) we find the conformal dimension of the FSSG models: $\Delta=\beta^{2} / 8 \pi+1-1 / L$.

In the infrared limit the groundstate energy of the massive model expands in powers of $e^{-M R}$ [15]. Adapting the arguments of [15,29] we obtain from the NLIEs

$$
E(R) \sim-4 \cos \frac{\pi}{L+2} \cos \pi \alpha \int \frac{d \theta}{2 \pi} \cosh \theta e^{-M R \cosh \theta} .
$$

With the choices of $L$ and $\alpha$ as given in the text this agrees perfectly with TBA results obtained for the perturbed cosets $\mathcal{M}_{L, K}+\phi_{\text {id,id,adj }}$ [22], the $\Phi_{13}$ perturbation of the $N=2$ supersymmetric minimal models [16] and the $S U(2)$ Gross-Neveu model [4].

We now test the NLIEs numerically, comparing against the effective central charge found using various TBA equations [22, 20, 16, 17]. These TBA equations correspond either to $\mathcal{M}_{L, K}+\phi_{\text {id,id,adj }}$ for integer $K$, or to the FSSG model at the specific coupling given in (5D). We also compare against the magnonic TBA system based on $\left(A_{1} \diamond T_{n}\right)_{L}$, identified in [17]

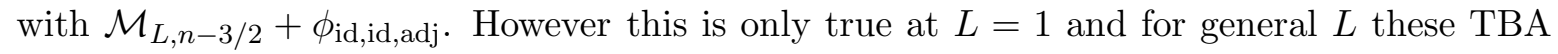


equations correspond to $\mathcal{M}_{L, n-L-1 / 2}+\phi_{\text {id,id,adj }}$. As no data for the chosen cases has previously been reported in the literature we solved both the TBA and NLIE equations numerically, using an iterative method. We checked stability of the solution by varying the number of iterations and the integration step size. The results are shown in table 2 . The tables include a diagrammatic version of the TBA system with a solid circle representing the massive node. We also reproduce one massless flow whose TBA diagram has crosses to indicate the nodes on which the massless driving terms are placed. In all cases the agreement is very good.

\begin{tabular}{|c|c|c|c|c|c|}
\hline Model & TBA & $\begin{array}{c}\text { NLIE } \\
(L, \xi, \alpha)\end{array}$ & $r$ & $\begin{array}{c}\text { TBA } \\
c_{\text {eff }}(r)\end{array}$ & $\begin{array}{l}\text { NLIE } \\
c_{\text {eff }}(r)\end{array}$ \\
\hline $\mathcal{M}_{2,4}+\phi_{\mathrm{id}, \mathrm{id}, \mathrm{adj}}$ & - $-\infty-\infty$ & $\left(4,4, \pm \frac{1}{4}\right)$ & $\begin{array}{l}1 \mathrm{E}-05 \\
100\end{array}$ & $\begin{array}{l}1.2499968080510 \\
1.0002668224816\end{array}$ & $\begin{array}{l}1.2499968080550 \\
1.0002668224816\end{array}$ \\
\hline $\mathcal{M}_{3, \frac{3}{2}}+\phi_{\text {id,id,adj }}$ & $\infty-\infty-a-\infty$ & $\left(3, \frac{7}{2}, \pm \frac{1}{7}\right)$ & $\begin{array}{l}0.01 \\
0.3 \\
\end{array}$ & $\begin{array}{l}1.5789577694079 \\
1.3491110222344 \\
\end{array}$ & $\begin{array}{l}1.5789577694082 \\
1.3491110222443 \\
\end{array}$ \\
\hline $\mathcal{M}_{3,4}+\phi_{\mathrm{id}, \mathrm{id}, \mathrm{adj}}$ & a-O-a-a-a-o & $\left(3,6, \pm \frac{1}{6}\right)$ & $\begin{array}{l}0.01 \\
0.3 \\
\end{array}$ & $\begin{array}{l}1.4590240568136 \\
1.2821840541083 \\
\end{array}$ & $\begin{array}{l}1.4590240568137 \\
1.2821840541085 \\
\end{array}$ \\
\hline$S_{2} \otimes S_{\mathrm{SG}}\left(\frac{3}{10}\right)$ & & $(2,3,0)$ & $\begin{array}{l}0.01 \\
0.3 \\
\end{array}$ & $\begin{array}{l}1.4889948011771 \\
1.2975198090220\end{array}$ & $\begin{array}{l}1.4889948011793 \\
1.2975198092226 \\
\end{array}$ \\
\hline$S_{4} \otimes S_{\mathrm{SG}}\left(\frac{1}{20}\right)$ & & $(4,1,0)$ & $\begin{array}{l}0.01 \\
0.3\end{array}$ & $\begin{array}{l}1.9854196180142 \\
1.7223919114950\end{array}$ & $\begin{array}{l}1.9854196180142 \\
1.7223919114948\end{array}$ \\
\hline
\end{tabular}

Table 2: Comparison of $c_{\text {eff }}(r)$ calculated using the TBA equations and the NLIE.

We can also enquire to which models the NLIEs based on $\left(A_{1} \diamond A_{L}\right)_{j}^{[1]}$ correspond. If we set the $j^{\text {th }}$ driving term to $-r \cosh \theta$ and all others to zero, the ultraviolet effective central charge becomes

$$
c_{\text {eff }}(0)=\frac{3(L-j+1)}{L-j+3}-\frac{6 \xi \alpha^{2}(L-j+1)}{(\xi+L)(\xi+j-1)} .
$$

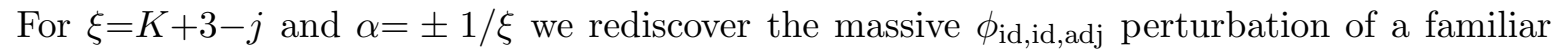
model: $\mathcal{M}_{L-j+1, K}$.

\section{$4 \quad N=2$ supersymmetric sine-Gordon model}

Recent interest in the $N=2$ supersymmetric sine-Gordon model [48] has arisen in two different contexts, both related to string theory. Though little is currently known about the excited state spectrum of the $N=2$ sine-Gordon model it is predicted to provide nontrivial information on the spectrum of states of closed superstrings in certain backgrounds 49. Also, boundary versions of the $N=2 \mathrm{SSG}$ model (see for example [50,51]) are relevant for open string theory.

The S-matrix of the $N=2$ SSG model has the usual factorised form [52, 9, 53, 16]

$$
S_{S G}^{N=2}(\tilde{\beta})=S^{N=2} \otimes S_{\mathrm{SG}}(\beta) .
$$

The supersymmetric piece $S^{N=2}$ is the sine-Gordon S-matrix at its $N=2$ supersymmetric point $\beta^{2} / 8 \pi=2 / 3$. The bosonic piece is the sine-Gordon S-matrix at coupling $\beta$. The $N=2$ coupling constant $\tilde{\beta}$ is related to the sine-Gordon coupling via [52]

$$
\tilde{\beta}^{2}=\beta^{2} /\left(1-\beta^{2} / 8 \pi\right)
$$


TBA equations derived from the $S^{N=2}$ S-matrix are encoded on $\left(A_{1} \diamond A_{3}\right)_{2}$ [16. As noted in 24, the TBA equations for the $N=2$ SSG model can, in theory, be obtained by gluing the $\left(A_{1} \diamond A_{3}\right)_{2}$ TBA system to that of the sine-Gordon model at coupling $\beta$. In practice this has been done only for $\tilde{\beta}^{2} / 8 \pi=k+1$, since at these points the SG TBA reduces to a simple Dynkin diagram: $D_{k+2}$. A second set of simple TBA equations exist for some related models: the minimal $N=2$ conformal field theories perturbed by the superfield $\Phi_{13}[16$.

To describe the groundstate energy of the $N=2$ SSG model at any coupling we replace the complicated sine-Gordon TBA equations with a single nonlinear integral equation, as we did above for the FSSG models. We propose equations similar to (131415) but of type $\left(A_{1} \diamond D_{3}\right)_{1}^{[1]}$ with $\alpha=0$, rather than $\left(A_{1} \diamond A_{L}\right)_{1}^{[1]}$. The nodes of $D_{n}$ are labelled such that 1 is the first node of the tail, and $n, n-1$ label the fork nodes. The stationary values $x_{a}^{0}$ are all infinite, which suggests that numerical solutions for very small $r$ will converge extremely slowly, if at all (this is equally true for any TBA equations with infinite stationary values). However combined with $x_{2}^{ \pm}=x_{3}^{ \pm}=1$ and $x_{1}^{ \pm}=0$ we find the correct ultraviolet central charge of 3. The leading logarithmic corrections to this value have been proposed for some of the models in 54]. It is simple to check the proposed NLIEs have the expected infrared limit.

If the mass is moved onto either of the magnonic nodes so that the NLIEs are of type $\left(A_{1} \diamond D_{3}\right)_{2}^{[1]}$, we find the effective central charge of the sausage models $S S T_{\lambda}^{+}$[55]. Again the possibly infinite set of TBA equations are in principle known. The simplest, occurring at $\lambda=1 / M$, are represented by an extended $D_{M}$ Dynkin diagram [55. A match is found with the NLIEs if we set $\xi=M-2$ and $\alpha=0$. Alternatively if the twist $\alpha$ is set to $\pm 1 /(M-2)$ we find the $\psi_{1} \bar{\psi}_{1}+\psi_{1}^{\dagger} \bar{\psi}_{1}^{\dagger}$ perturbation of the $Z_{M-2}$ models, usually denoted $H_{M-2}^{(0)}$ [56, 19]. The models denoted $H_{M-2}^{(\pi)}$ refer to the massless perturbation of the $Z_{M-2}$ models. The flow of the effective central charge from these models to the minimal models $\mathcal{M}(M-1, M)$ can be reproduced within the NLIEs provided the magnonic nodes are given driving terms of the form $-\frac{1}{2} r e^{\theta}$ and $-\frac{1}{2} r e^{-\theta}$. Upon resetting $\alpha$ to zero we find the effective central charge of the 'massless' sausage models $S S T_{1 / M}^{-}$.

Table 3 shows a numerical comparison of results found using this second set of NLIEs against some of the simplest TBA systems.

\begin{tabular}{|c|c|c|c|c|c|}
\hline Model & TBA & $\begin{array}{l}\text { NLIE } \\
(\xi, \alpha)\end{array}$ & $r$ & $\begin{array}{c}\mathrm{TBA} \\
c_{\mathrm{eff}}(r)\end{array}$ & $\begin{array}{l}\text { NLIE } \\
c_{\text {eff }}(r)\end{array}$ \\
\hline$S^{N=2} \otimes S_{S G}\left(\frac{3}{4}\right)$ & & $(3,0)$ & $\begin{array}{l}0.01 \\
0.3\end{array}$ & $\begin{array}{l}2.5646432734143 \\
1.9298961954447\end{array}$ & $\begin{array}{l}2.5646432734187 \\
1.9298961954452\end{array}$ \\
\hline$S S T_{1 / 4}^{+}$ & o- & $(2,0)$ & \begin{tabular}{|l}
0.01 \\
0.3 \\
\end{tabular} & $\begin{array}{l}1.7401548125701 \\
1.3902395891608\end{array}$ & $\begin{array}{l}1.7401548125703 \\
1.3902395891609\end{array}$ \\
\hline$H_{5}^{(0)}$ & & $\left(5, \pm \frac{1}{5}\right)$ & $\begin{array}{l}0.01 \\
0.3 \\
\end{array}$ & $\begin{array}{l}1.1373080354277 \\
1.0190548066399\end{array}$ & $\begin{array}{l}1.1373080354327 \\
1.0190548066674\end{array}$ \\
\hline$H_{4}^{(\pi)}$ & $\begin{aligned} \phi \\
\phi\end{aligned}$ & $\left(4, \pm \frac{1}{4}\right)$ & $\begin{array}{l}1 \mathrm{E}-05 \\
100\end{array}$ & $\begin{array}{l}0.9999980062005 \\
0.8003485312633\end{array}$ & $\begin{array}{l}0.9999980062002 \\
0.8003485312633\end{array}$ \\
\hline
\end{tabular}

Table 3: Comparison of $c_{\text {eff }}(r)$ found using TBA equations and NLIEs of type $\left(A_{1} \diamond D_{3}\right)_{a}^{[1]}$. 


\section{Summary}

The SS model [54] is a two-parameter family of integrable models whose name follows from the S-matrix, which is a direct product of two sine-Gordon S-matrices. All of the models discussed above can be found as an SS model at specific values of the parameters [54,57. We leave for future investigation the possibility of gluing two sine-Gordon NLIEs together to describe the groundstate energy of this family of models.

Motivated by the existing results for the sine-Gordon model both sets of new NLIE should be generalised to encode the full finite size spectrum of the associated models. Furthermore, it is curious that the TBA driving term trick to find equations for the massless flows also works for the NLIEs; though only for the unitary coset models. A rather different method was used in [39] for the sine-Gordon model, also [43, 58, to obtain NLIEs describing massless flows at any value of $\beta^{2}$. It remains to be seen whether a similar idea can be used on the new NLIEs. Finally it would be extremely interesting to develop the finite size effects of the fractional sine-Gordon models defined on a half-line.

Acknowledgments - I'm grateful to Zoltan Bajnok, Andreas Klümper, Paul Pearce, Francesco Ravanini and Gerard Watts for useful discussions, and to Patrick Dorey and Roberto Tateo for useful suggestions and discussions. I thank the EPSRC for a Postdoctoral Fellowship and the Department of Mathematics and Statistics, University of Melbourne for hospitality.

\section{References}

[1] P. Di Vecchia and S. Ferrara, 'Classical solutions in two-dimensional supersymmetric field theories', Nucl. Phys. B130 (1977) 93

[2] J. Hruby, 'On the supersymmetric sine-Gordon model and a two dimensional 'bag", Nucl. Phys. B131 (1977) 275

[3] S. Ferrara, L. Giradello and S. Sciuto, 'An infinite set of conservations laws of the supersymmetric sine-Gordon theory', Phys. Lett. B76 (1978) 303-306

[4] L. Giradello and S. Sciuto, 'Inverse scattering-like problem for supersymmetric models', Phys. Lett. B77 (1978) 267

[5] I. Yamanaka and R. Sasaki, 'Supervirasoro algebra and solvable supersymmetric quantum field theories', Prog. Theory. Phys. 79 (1988) 1167

[6] D. Bernard and A. LeClair, 'Residual quantum symmetries of the restricted sine-Gordon theories', Nucl. Phys. B340 (1990) 721-751

[7] A.B. Zamolodchikov, 'Fractional-spin integrals of motion in perturbed conformal field theory', Beijing 1989, Proceedings, Field, string and quantum gravity. 349-372

[8] C. Ahn, D. Bernard and A. LeClair, 'Fractional supersymmetries in perturbed coset CFTs and integrable soliton theory', Nucl. Phys. B346 (1990) 409-439

[9] D. Bernard and A. LeClair, 'The fractional supersymmetric sine-Gordon models', Phys. Lett. B247 (1990) 309-316

[10] F.A. Smirnov, 'Reductions of the sine-Gordon model as a perturbation of minimal models of conformal field theory', Nucl. Phys. B337 (1990) 156-180

[11] A. LeClair, 'Restricted sine-Gordon theory and the minimal conformal series', Phys. Lett. B230 (1989) 103-107 
[12] A.B. Zamolodchikov and Al.B. Zamolodchikov, 'Factorized S matrices in two dimensions as the exact solutions of certain relativistic field models', Ann. of Phys. 120 (1979) 253-291

[13] K. Schoutens, 'Supersymmetry and factorizable scattering', Nucl. Phys. B344 (1990) $665-695$

[14] C.N. Yang and C.P. Yang, 'Thermodynamics of one-dimensional systems of bosons with repulsive delta function interaction', J. Math. Phys. 10 (1969) 1115-1122

[15] Al.B. Zamolodchikov, 'Thermodynamic Bethe Ansatz in relativistic models: Scaling 3-state Potts and Lee-Yang models', Nucl. Phys. B342 (1990) 695-720

[16] P. Fendley and K. Intriligator, 'Scattering and thermodynamics of fractionally charged supersymmetric solitons', Nucl. Phys. B372 (1992) 533-558

[17] F. Ravanini, R. Tateo and A. Valleriani, 'Dynkin TBAs', Int. J. Mod. Phys. A8 (1993) $1707-1727$

[18] F. Ravanini, R. Tateo and A. Valleriani, 'A new family of diagonal A-D-E related scattering theories', Phys. Lett. B293 (1992) 361-366

[19] V.A. Fateev and Al.B. Zamolodchikov, 'Integrable perturbations of $\mathbb{Z}_{N}$ parafermion models and the $O(3)$ sigma model', Phys. Lett. B271 (1991) 91-100

[20] R. Tateo, 'The sine-Gordon model as $\frac{\mathcal{S O}(n)_{1} \times \mathcal{S O}(n)_{1}}{\mathcal{S O}(n)_{2}}$ perturbed coset theory and generalizations', Int. J. Mod. Phys. A10 (1995) 1357-1376

[21] R. Tateo, 'New functional dilogarithm identities and sine-Gordon Y-systems', Phys. Lett. B355 (1995) 157-164

[22] Al.B. Zamolodchikov, 'TBA equations for integrable perturbed $S U(2)_{k} \times S U(2)_{l} / S U(2)_{k+l}$ coset models', Nucl. Phys. Lett. B366 (1991) 122-132

[23] C. Ahn, 'Thermodynamics and Form Factors of Supersymmetric Integrable Field theories', Nucl. Phys. B422 (1994) 449-475

[24] P. Fendley and K. Intriligator, 'Scattering and Thermodynamics in integrable $N=2$ theories', Nucl. Phys. B380 (1992) 265-292

[25] E. Melzer, 'Supersymmetric analogs of the Gordon-Andrews identities, and related TBA systems', hep-th/9412154

[26] M. Moriconi and K. Schoutens, 'Thermodynamic Bethe Ansatz for $N=1$ Supersymmetric Theories', Nucl. Phys. B464 (1996) 472-491

[27] Al.B. Zamolodchikov, 'On the thermodynamic Bethe ansatz equations for the reflectionless ADE scattering theories', Phys. Lett. B253 (1991) 391-394

[28] A. Kuniba, K. Sakai and J. Suzuki, 'Continued fraction TBA and functional relations in XXZ model at root of unity', Nucl. Phys. B525 (1998) 597-626

[29] C. Destri and H.J. de Vega, 'New thermodynamic Bethe ansatz equations without strings', Phys. Rev. Lett. 69 (1992) 2313-2317

[30] G. Feverati, F. Ravanini and G. Takacs, 'Nonlinear Integral Equation and Finite Volume Spectrum of Sine-Gordon Theory', Nucl. Phys. B540 (1999) 543-586

[31] G. Feverati, F. Ravanini and G. Takacs, 'Scaling functions in the odd charge sector of sine-Gordon/ massive Thirring theory', Phys. Lett. B444 (1998) 442-450 
[32] A. Klümper, M.T. Batchelor and P.A. Pearce, 'Central charges of the 6- and 19-vertex models with twisted boundary conditions', J. Phys. A24 (1991) 3111-3133

[33] P.A. Pearce and A. Klümper, 'Finite-Size Corrections and Scaling Dimension of Solvable Lattice Models: An Analytic Method', Phys. Rev. Lett. 66 (1991) 974-977

[34] J. Suzuki, 'Spinons in magnetic chains of arbitrary spins at finite temperatures', J. Phys. A32 (1999) 2341-2359

[35] A.B. Zamolodchikov, 'Renormalization group and perturbation theory about fixed points in two-dimensional field theory', Sov. J. Nucl. Phys. 46 (1987) 1090-1096

[36] A.B. Zamolodchikov, 'Integrable field theory from conformal field theory', Adv. Stud. in Pure Maths. 19 (1989) 641-674

[37] Al.B. Zamolodchikov, 'Thermodynamic Bethe Ansatz for RSOS scattering theories', Nucl. Phys. B358 (1991) 497-523

[38] Al.B. Zamolodchikov, 'From tricritical Ising to critical Ising by Thermodynamic Bethe Ansatz', Nucl. Phys. B358 (1991) 524-546

[39] Al.B. Zamolodchikov, 'Thermodynamics of imaginary coupled sine-Gordon: Dense polymer finite-size scaling function', Phys. Lett. B335 (1994) 436-443

[40] Al.B. Zamolodchikov, 'Painleveé III and 2D Polymers', Nucl. Phys. B432 (1994) $427-456$

[41] D. Fioravanti, A. Mariottini, E. Quattrini and F. Ravanini, 'Excited state Destri-De Vega equation for sine-Gordon and restricted sine-Gordon models', Phys. Lett. B390 (1997) 243-251

[42] G. Feverati, F. Ravanini and G. Takacs, 'Nonlinear integral equation and finite volume spectrum of minimal models perturbed by $\phi_{1,3}$ ', Nucl. Phys. B570 (2000) 615-643,

[43] P. Dorey, C. Dunning and R. Tateo, 'New families of flows between two-dimensional conformal field theories', Nucl. Phys. B578 (2000) 699-727

[44] T.R. Klassen and E. Melzer, 'Purely elastic scattering theories and their ultraviolet limits', Nucl. Phys. B338 (1990) 485-528

[45] A.N. Kirillov, 'Dilogarithm identities', Prog. Theor. Phys. Suppl. 118 (1995) 61-142

[46] P. Dorey and R. Tateo, 'Differential equations and integrable models: the $S U(3)$ case', Nucl. Phys. B571 (2000) 583-606

[47] P. Fendley, 'Integrable sigma models and perturbed coset models', JHEP 0105 (2001) 050

[48] K. Kobayashi and T. Uematsu, ' $N=2$ supersymmetric sine-Gordon theory and conservation laws', Phys. Lett. B264 (1991) 107-13

[49] J. Maldacena and L. Maoz, 'Strings on pp-waves and massive two dimensional field theories', hep-th/0207284

[50] N.P. Warner, 'Supersymmetry in boundary integrable quantum field theories', Nucl. Phys. B450 (1995) 663-694

[51] R.I. Nepomechie, 'Boundary S matrices with $N=2$ supersymmetry', Phys. Lett. B516 (2001) 161-164 ; 'The boundary $N=2$ supersymmetric sine-Gordon model', Phys. Lett. B516 (2001) 376-382 
[52] K. Kobayashi and T. Uematsu, 'S matrix of $N=2$ Supersymmetric Sine-Gordon model', Phys. Lett. B275 (1992) 361-370 ; 'Quantum conserved charges and S matrices in $N=2$ supersymmetric sine-Gordon theory', Prog. Theor. Phys. Suppl. 110 (1992) $347-364$

[53] P. Fendley, S.D. Mathur, C. Vafa and N.P. Warner, 'Integrable deformations and scattering matrices for the $N=2$ supersymmetric discrete series ', Phys. Lett. B243 (1990) 257-264

[54] V.A. Fateev, 'The sigma model (dual) representation for a two-parameter family of integrable quantum field theories', Nucl. Phys. B473 (1996) 509-538

[55] V.A. Fateev, E. Onofri and Al.B. Zamolodchikov, 'Integrable deformations of the $O(3)$ sigma model. The sausage model', Nucl. Phys. B406 (1993) 521-565

[56] V.A. Fateev, 'Integrable deformations in $Z_{n}$-symmetrical models of the conformal quantum field theory', Int. J. Mod. Phys. A6 (1991) 2109-2132

[57] P. Baseilhac and V.A. Fateev, 'Expectation values of local fields for a two-parameter family of integrable models and related perturbed conformal field theories', Nucl. Phys. B532 (1998) 567-587

[58] C. Dunning, 'Massless flows between minimal $W$ models', Phys. Lett. B537 (2002) 297-305 